\title{
List of Participants of IAU Symposium No.156
}

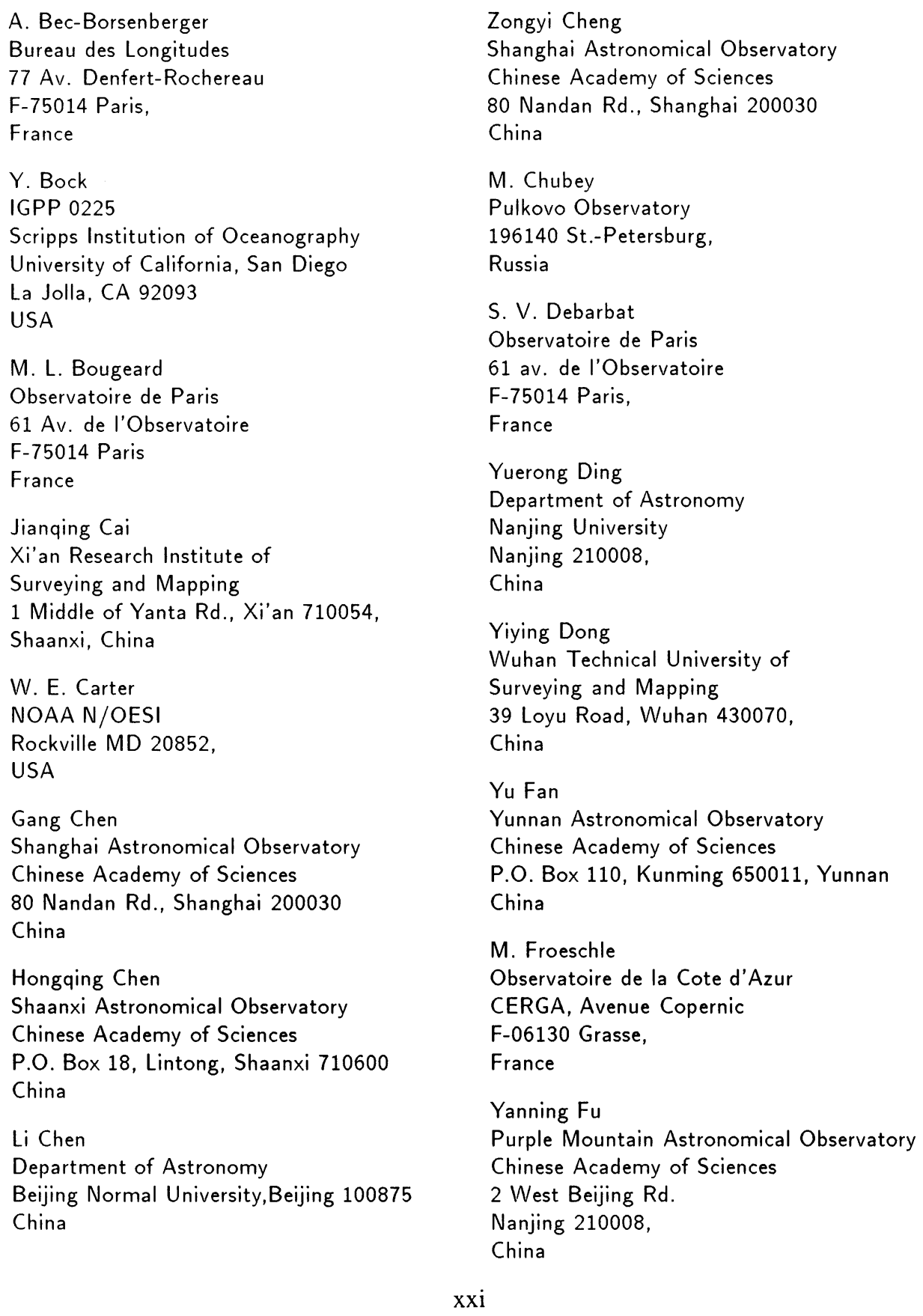

\author{
Zongyi Cheng \\ Shanghai Astronomical Observatory \\ Chinese Academy of Sciences \\ 80 Nandan Rd., Shanghai 200030 \\ China
}

M. Chubey

Pulkovo Observatory

196140 St.-Petersburg,

Russia

S. V. Debarbat

Observatoire de Paris

61 av. de l'Observatoire

F-75014 Paris,

France

Yuerong Ding

Department of Astronomy

Nanjing University

Nanjing 210008,

China

Yiying Dong

Wuhan Technical University of

Surveying and Mapping

39 Loyu Road, Wuhan 430070 ,

China

Yu Fan

Yunnan Astronomical Observatory

Chinese Academy of Sciences

P.O. Box 110, Kunming 650011, Yunnan

China

M. Froeschle

Observatoire de la Cote d'Azur

CERGA, Avenue Copernic

F-06130 Grasse,

France

Yanning $\mathrm{Fu}$

Purple Mountain Astronomical Observatory

Chinese Academy of Sciences

2 West Beijing Rd.

Nanjing 210008,

China 
Buxi Gao

Institute of Geodesy and Geophysics

Chinese Academy of Sciences

54 Xudong Rd., Wuchang 430077 ,

Hubei,

China

Jie Gao

Department of Astronomy

Nanjing University

Nanjing 210008,

China

Shumo Gong

Purple Mountain Astronomical Observatory

Chinese Academy of Sciences

2 West Beijing Rd.,

Nanjing 210008,

China

E. Groten

Technical University Darmstade

Institute of Physical Geodesy

Petersenstr. 13,

D-6100 Darmstadt

Germany

Xingjun Guan

$X_{i}$ an Research Institute

of Surveying and Mapping

1 Middle of Yanta Rd., Xi'an 710054,

Shaanxi, China

\section{V.S. Gubanov}

Institute of Applied Astronomy

Russian Academy of Sciences

197022 Zhdanov, Str. 8

St.-Petersburg

Russia

Tangyong Guo

Institute of Seismology

State Seismological Bureau of China

Xiao Hong Shan 70, Wuchang, Wuhan

430071,

Hubei,

China
Chunhao Han

Department of Geodesy

Zhengzhou Institute of

Surveying and Mapping

Zhengzhou 450052,

China

Miaofu $\mathrm{He}$

Shanghai Astronomical Observatory

Chinese Academy of Sciences

80 Nandan Rd.,

Shanghai 200030

China

D. Hestroffer

Bureau des Longitudes

77 Avenue, Denfert-Rochereau

F-75014 Paris,

France

E. Hoeg

University Observatory

1350 Copenhagen K,

Denmark

Yingmin Hua

Shanghai Astronomical Observatory

Chinese Academy of Sciences

80 Nandan Rd., Shanghai 200030

China

Cheng Huang

Shanghai Astronomical Observatory

Chinese Academy of Sciences

80 Nandan Rd., Shanghai 200030

China

P. lanna

Leander McCormick Observatory

University of Virginia

Charlottesville VA 22903-0818

USA

M. Inoue

Nobeyama Radio Observatory

National Astronomical Observatory

Nobeyama, Minamisaku

Nagano 384-13,

Japan 
C. S. Jacobs

Jet Propulsion Laboratory

$\mathrm{M} / \mathrm{S} 238-700$

4800 Oak Grove Drive

Pasadena CA 91109,

USA

H. Jahreiss

Astronomisches Rechen-Institut

Moenchhofstr. 12-14

D-6900 Heidelberg

Germany

Desheng Ji

Shanghai Asronomical Observatory

Chinese Academy of Sciences

80 Nandan Rd.,

Shanghai 200030

China

Wenjing Jin

Shanghai Astronomical Observatory

Chinese Academy of Sciences

80 Nandan Rd.,

Shanghai 200030

China

N. Kharchenko

Main Astronomical Observatory

Ukranian Academy of Sciences,

$252127 \mathrm{Kiev}$

Ukraine

B. Kolaczek

Space Research Center

Bartycka 18A, 00716 Warsaw

Poland

Yi Kong

Shanghai Astronomical Observatory

Chinese Academy of Sciences

80 Nandan Rd., Shanghai 200030

China

J. Kovalevsky

Observatoire de la Cote d'Azur

CERGA, Avenue Copernic

F-06130 Grasse

France
Qi Li

Beijing Astronomical Observatory

Chinese Academy of Sciences

Zhongguan Cun, Beijing 100080 ,

China

Yulin Li

Research Institute of

Surveying and Mapping

State Surveying and Mapping Bureau

16 Northern Taiping Rd.

Beijing 100039,

China

Zhenghang $\mathrm{Li}$

Wuhan Technical University of

Surveying and Mapping

39 Loyu Road, Wuhan 430070

China

Zhengxin $\mathrm{Li}$

Shanghai Astronomical Observatory

Chinese Academy of Sciences

80 Nandan Rd., Shanghai 200030

China

Zhian Li

Department of Astronomy

Beijing Normal University

Beijing 100875

China

Zhigang Li

Shaanxi Astronomical Observatory

Chinese Academy of Sciences

P.O. Box 18, Lintong, Shaanxi 710600 ,

China

Haiqi Liang

Shanghai Astronomical Observatory

Chinese Academy of Sceinces

80 Nandan Rd., Shanghai 200030

China

Dechun Liao

Shanghai Astronomical Observatory

Chinese Academy of Sciences

80 Nandan Rd., Shanghai 200030

China 
xxiv

J. H. Lieske

Jet Propulsion Laboratory

$\mathrm{M} / \mathrm{S}$ 301-150

4800 Oak Grove Drive

Pasadena, CA 91109,

USA

Qinchang Lin

Guangzhou Satellite Station

Wushan, Guangzhou 510640,

China

Quanbao Ling

Shanghai Astronomical Observatory

Chinese Academy of Sceinces

80 Nandan Rd., Shanghai 200030

China

Ciyuan Liu

Shaanxi Astronomical Observatory

Chinese Academy of Sciences

P.O. Box 18, Lintong

Shaanxi 710600

China

S. Lowe

Jet Propulsion Laboratory,

$\mathrm{M} / \mathrm{S}$ 238-700

4800 Oak Grove Drive

Pasadena, CA 91109

USA

Chunlin Lu

Purple Mountain Astronomical Observatory

Chinese Academy of Sciences

2 West Beijing Rd., Nanjing 210008

China

Dingjiang Luo

Beijing Astronomical Observatory

Chinese Academy of Sciences

Zhongguan Cun, Beijing 100080,

China

Anxin $\mathrm{Ma}$

Shaanxi Astronomical Observatory

Chinese Academy of Sciences

P.O. Box 18, Lintong, Shaanxi 710600

China
Chopo $\mathrm{Ma}$

NASA

GSFC, Code 926.9

Greenbelt MD 20771

USA

Ailin Mao

Shanghai Astronomical Observatory

Chinese Academy of Sciences

B80 Nandan Rd.,

Shanghai 200030

China

Xinjie Mao

Department of Astronomy

Beijing Normal University

Beijing 100875

China

D. McCarthy

U.S. Naval Observatory

34th \& Mass, Ave. NW

Washington D.C. 20392

USA

M. Miyamoto

National Astronomical Observatory

Osawa, Mitaka

Tokyo 181,

Japan

B. Morando

Bureau des Longitudes

77 Avenue,

Denfert-Rochereau

F-75014 Paris,

France

I. I. Mueller

Geodetic Science \& Surveying

Ohio State University

Columbus $\mathrm{OH}$ 43210-1247,

USA

L. Mureddu

Stazione Astronomica-via Ospedale 72

Cagliari 09124,

Italy 
Zhaoming Nie

Yunnan Astronomical Observatory

Chinese Academy of Sciences

P.O. Box 110, Kunming 650011, Yunnan

China

P. Paquet

Royal Observatory of Belgium

Av. Circulaire 3

1180 Brussels.

Belgium

P. Park

Korea Astronomy Observatory

36-1 Whaamdong, Yuseonggu

Daejeon 305-348,

Korea

I. Petrovskaya

Astronomical Observatory

Petersburg University

St. Petersburg, 198904,

Russia

G. Pinigin

Nikolaev Astronomical Observatory

Observatornaya 1

327030 Nikolaev

Ukraine

Baochen Qian

Shanghai Astronomical Observatory

Chinese Acadmy of Sciences

80 Nandan Rd.,

Shanghai 200030

China

Zhihan Qian

Shanghai Astronomical Observatory

Chinese Academy of Sciences

80 Nandan Rd.,

Shanghai 200030

China

Xu Ren

Shanghai Astronomical Observatory

Chinese Academy of Sciences

80 Nandan Rd., Shanghai 200030

China
J. Reynolds

ATNF

P.O. Box 76

Epping, NSW 2121

Australia

L.V. Rykhlova

Institute of Astronomy

Russian Academy of Sciences

Pjatnitskaja Str. 48,

109017, Moscow

Russia

E. Schilbach

Universitat Potsdam

Sternwarte Babelsberg

R.-Luxemburg-Str. 17a

0-1591 Potsdam

Germany

R.-D. Scholz

Universitat Potsdam

Sternwarte Babelsberg

R.-Luxemburg-Str. 17a

0-1591 Potsdam

Germany

H. Schwan

Astronomisches Rechen-Institut

Monchhofstr. 12-14

D-6900 Heidelberg

Germany

P. K. Seidelmann

US Naval Observatory

34th \& Mass. Ave. NW

Washington D.C. 20392

USA

N. Sekiguchi

Musashidai 3-16-8 Fuchu

Tokyo 183,

Japan

O. E. Shornikov

Astrometrical Laboratory of the Tatarstan Scientific Academy Kazan, 420018,

Russia 
M. Soma

National Astronomical Observatory

Osawa, Mitaka,

Tokyo 181

Japan

R. Stone

U.S. Naval Observatory, Flagstaff Station

P.O. Box 1149

Flagstaff AZ 86002

USA

\section{K. A. Strand}

3200 Rowland PL. NW

Washington D.C. 20008,

USA

L. Taff

Space Telescope Science Institute 3700 San Martin Drive

Baltimore MD 21218,

USA

Y. Takahashi

Communication Research Laboratory

Kashima Space Research Center

893-1, Hirai, Kashima,

Ibaraki, 314

Japan

W. Tarady

Main Astronomical Observatory

Ukranian Academy of Sciences

$252127 \mathrm{Kiev}$

Ukraine

Kaiping Tian

Shanghai Astronomical Observatory

Chinese Academy of Sciences

80 Nandan Rd.,

Shanghai 200030

China

J. Vondra'k

Astronomical Institute

Czechoslovakia Academy of Sciences

Budecska 6

12023 Praha 2

Czechoslovakia
H. G. Walter

Astronomisches Rechen-Institut

Monchhofstr. 12-14, D-6900 Heidelberg

Germany

Lai Wan

Shanghai Astronomical Observatory

Chinese Academy of Sciences

80 Nandan Rd., Shanghai 200030

China

Hongqi Wang

Shaanxi Astronomical Observatory

Chinese Academy of Sciences

P.O. Box 18, Lintong

Shaanxi 710600,

China

Jiaji Wang

Shanghai Astronomical Observatory

Chinese Academy of Sciences

80 Nandan Rd., Shanghai 200030

China

Qi Wang

Institute of Seismology

State Seismological Bureau of China

Xiao Hong Shan 70, Wuchang, Wuhan 430071, Hubei,

China

Shuhe Wang

Shanghai Astronomical Observatory

Chinese Academy of Sciences

80 Nandan Rd., Shanghai 200030

China

Zhengming Wang

Shaanxi Astronomical Observatory

Chinese Academy of Sciences

P.O. Box 18, Lintong, Shaanxi 710600

China

Xue Wei

Shanghai Astronomical Observatory

Chinese Academy of Sciences

80 Nandan Rd.,

Shanghai 200030

China 
Ziqin Wei

Xi'an Research Institute of

Surveying and Mapping

1 Middle of Yanta Rd., Xi'an 710054,

Shaanxi,

China

Bin Wu

Institute of Geodesy and Geophysics

Chinese Academy of Sciences

54 Xudong Rd., Wuchang 430077, Hubei

China

Huaiwei Wu

Shanghai Astronomical Observatory

Chinese Academy of China

80 Nandan Rd., Shanghai 200030

China

Xinghua $\mathrm{Wu}$

Institute of Seismology

State Seismological Bureau of China

Xiao Hong Shan 70, Wuchang

Wuhan 430071, Hubei

China

Hanlin Xia

$X_{i}$ an Research Institute of

Surveying and Mapping

1 Middle of Yanta Rd., Xi'an 710054,

Shaanxi,

China

Yifei Xia

Department of Astronomy

Nanjing University

Nanjing 210008

China

Naiyuan Xiao

Department of Astronomy

Nanjing University,

Nanjing 210008,

China

BangXin Xu

Department of Astronomy

Nanjing University, Nanjing 210008,

China
Huaguan Xu

Shanghai Astronomical Observatory

Chinese Academy of Sciences

80 Nandan Rd., Shanghai 200030

China

Jiayan Xu

Shaanxi Astronomical Observatory

Chinese Academy of Sciences

P.O. Box 18, Lintong

Shaanxi, 710600 ,

China

Tongqi $X u$

Shanghai Astronomical Observatory

Chinese Academy of Sciences

80 Nandan Rd., Shanghai 200030

China

Fumin Yang

Shanghai Astronomical Observatory

Chinese Academy of Sciences

80 Nandan Rd., Shanghai 200030

China

Zhigen Yang

Shanghai Astronomical Observatory

Chinese Academy of Sciences

80 Nandan Rd., Shanghai 200030

China

Baoan Yao

Shanghai Astronomical Observatory

Chinese Academy of Sciences

80 Nandan Rd., Shanghai 200030

China

Zhenguo Yao

Astrometry Department

US Naval Observatory

34th \& Mass. Ave. NW

Washington, DC. 20392

USA

Ya.S. Yatskiv

Main Astronomical Observatory

Ukranian Academy of Sciences

$252127 \mathrm{Kiev}$

Ukraine 
xxviii

Shuhua Ye

Shanghai Astronomical Observatory

Chinese Academy of Sciences

80 Nandan Rd., Shanghai 2000030

China

M. Yoshizawa

National Astronomical Observatory

Osawa, Mitaka, Tokyo 181

Japan

Jianwei Zhang

Shanghai Astronomical Observatory

Chinese Academy of Sceinces

80 Nandan Rd., Shanghai 200030,

China

Jie Zhang

IGPP 0225 Scripps Institute of Oceanography

University of California, San Diego

La Jolla, CA 92093

USA

Gang Zhao

Shanghai Astronomical Observatory

Chinese Academy of Sciences

80 Nandan Rd.,

Shanghai 200030

China

Junliang Zhao

Shanghai Astronomical Observatory

Chinese Academy of Sciences

80 Nandan Rd.,

Shanghai 200030

China

Ming Zhao

Shanghai Astronomical Observatory

Chinese Academy of Sciences

80 Nandan Rd.,

Shanghai 200030

China

Dawei Zheng

Shanghai Astronomical Observatory

Chinese Academy of Sciences

80 Nandan Rd., Shanghai 200030

China
Xingwu Zheng

Department of Astronomy

Nanjing University

Nanjing 210008,

China

Yong Zheng

Shanghai Astronomical Observatory

Chinese Academy of Sciences

80 Nandan Rd., Shanghai 200030

China

Yaozhong Zhu

Institute of Geodesy and Geophysics

Chinese Academy of Sciences

54 Xudong Rd., Wuchang 430077, Hubei,

China

Zi Zhu

Shaanxi Observatory

Chinese Academy of Sciences

P.O. Box 18, Lintong

Shaanxi 710600 ,

China 